\title{
Increased Rho-kinase expression and activity and pulmonary endothelial dysfunction in smokers with normal lung
} function

\author{
S. Duong-Quy*, P. Dao*, T. Hua-Huy*, C. Guilluy ${ }^{\#}$, P. Pacaud ${ }^{\#}$ and A.T. Dinh-Xuan*
}

ABSTRACT: Endothelial dysfunction is one of the main consequences of the toxic effects of cigarette smoke on the vascular system. Increasing evidence suggests that the small G-protein RhoA and its downstream effectors, the Rho-kinases (ROCKs), are involved in systemic endothelial dysfunction induced by cigarette smoke. This study aimed to evaluate the role of the RhoA/ROCKs pathway in pulmonary artery endothelial function in current smokers with normal lung function.

Lung tissues were obtained from nonsmokers and smokers who underwent lobectomy for lung carcinoma. Arterial relaxation in response to acetylcholine (ACh) was assessed in isolated pulmonary arterial rings. Protein expressions and activities of endothelial nitric oxide synthase (eNOS), ROCKs and the myosin phosphatase subunit 1 (MYPT-1) were sought.

Relaxation in response to $\mathrm{ACh}$ was significantly lower in smokers as compared with nonsmokers ( $n=8$ in each group), consistent with reduced eNOS activity in the former compared with the latter. eNOS protein expression remained, however, the same in both groups. Expression of ROCKs, guanosine triphosphate-RhoA and phosphorylated MYPT-1 were significantly increased in smokers compared with controls.

Pulmonary endothelial dysfunction is present in smokers whose lung function has not yet been impaired. Reduced activity of eNOS accounts at least in part for this endothelial dysfunction. Increased expression and activity of ROCKs accounts for another part through direct or indirect inhibition of the Rho-A/ROCKs pathway on nitric oxide synthesis and sustained pulmonary vasoconstriction through inhibition of myosin phosphatase.

KEYWORDS: Cyclic guanosine monophosphate, endothelial dysfunction, endothelial nitric oxide synthase, RhoA, Rho-kinases, tobacco smoke

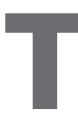

obacco smoke is associated with high mortality and morbidity related to cardiovascular disease [1], and is currently the leading cause of chronic obstructive pulmonary disease (COPD) [2]. The most prominent pathological changes in COPD patients are progressive airflow limitation, peripheral airway inflammation and pulmonary vascular remodelling $[3,4]$. Structural changes of the pulmonary vasculature, however, are not exclusive to patients with severe COPD, as they have been found in patients with milder degrees of airway obstruction, and even in heavy smokers without airflow limitation [5]. Together with its deleterious effects on peripheral airways, tobacco smoke also causes systemic endothelial dysfunction in active and passive smokers [6, 7], in whom endothelial dysfunction often precedes structural changes of pulmonary vessels $[8,9]$.

In smokers, endothelial dysfunction might directly result from the toxic effects of, or be indirectly due to, the release of inflammatory mediators induced by free radicals derived from tobacco smoke [10]. The precise mechanisms of tobacco smoke-related endothelial dysfunction are not fully understood, but it is hypothesised that decreased production or reduced bioavailability of nitric oxide (NO) in the pulmonary vasculature of smokers might be partly implicated [11].

Recent studies suggest that the small G-protein RhoA and its downstream effectors the Rhokinases (ROCK-1 or ROCK- $\beta$ and ROCK-2 or

\section{AFFILIATIONS}

*Université Paris Descartes, Faculté de Médecine, Assistance Publique Hôpitaux de Paris, Service de Physiologie - Explorations Fonctionnelles, Hôpital Cochin, Paris, and

\#INSERM, U915, Faculté des Sciences, Université de Nantes, Nantes, France.

CORRESPONDENCE

A.T. Dinh-Xuan

Service de Physiologie Explorations Fonctionnelles Hôpital Cochin

27 rue du Faubourg

Saint-Jacques

75104 Paris

France

E-mail: anh-tuan.dinh-xuan@

cch.aphp.fr

Received:

April 122010

Accepted after revision:

May 202010

First published online:

June 072010 
ROCK- $\alpha$ ) are also implicated in the pathogenesis of endothelial dysfunction [12]. In smooth muscle cells (SMCs), activation of Rho-kinases by guanosine triphosphate (GTP)-RhoA causes phosphorylation of the myosin-binding subunit of myosin phosphatase (MPT), also called myosin phosphatase subunit 1 (MYPT-1). The resulting inhibition of MPT prolongs actinmyosin interaction and sustains pre-existing vasoconstriction [13]. Furthermore, RhoA/Rho-kinase downregulates endothelial NO synthase (eNOS) expression and impairs NO production, thus reducing a potent counteracting mechanism to increased vascular tone [14].

Increased RhoA/Rho-kinase activity has been implicated in systemic endothelial dysfunction but evidence is still lacking regarding its role in pulmonary endothelial dysfunction. The aims of the present study were two-fold; first to assess the presence (or the absence) of impaired endothelium-dependent relaxation of pulmonary arteries in smokers with normal lung function and, secondly, to determine the respective contributions of reduced eNOS/NO and increased RhoA/Rho-kinase in tobacco smoke-related endothelial dysfunction.

\section{METHODS}

\section{Subjects and tissue preparations}

Patients who underwent lung resection for carcinoma were enrolled in the present study after giving informed consent, as approved by the local ethics committee (Comité de protection des personnes Ile de France, Paris, France). They were divided into two groups: nonsmokers and current smokers. All but two nonsmokers had never smoked. The two remaining subjects were ex-smokers (5 and 10 pack-yrs) but had quit smoking for 20 and 25 yrs, respectively. All patients had normal lung function, as defined by slow and forced vital capacity (FVC), total lung capacity and forced expiratory volume in $1 \mathrm{~s}$ (FEV1) $\geqslant 80 \%$ of predicted values. Absence of airflow obstruction was defined as a ratio of FEV1/FVC $\geqslant 0.7$ [15].

Peripheral lung tissues and proximal pulmonary arteries were carefully dissected from lobectomy tissue free from macroscopic signs of lung tumour. Rings of 2-3 mm external diameter and $4-6 \mathrm{~mm}$ of length were cut and immediately placed in cold Krebs-Henseleit solution $\left(4^{\circ} \mathrm{C}\right)$ for vasoreactivity study within the following $30 \mathrm{~min}$. The other specimens of pulmonary arterial and lung parenchyma were stored at $-80^{\circ} \mathrm{C}$ for Western blotting, or they were fixed overnight in $4 \%$ formalin and embedded in paraffin for immunohistochemistry assessment.

\section{Chemical products}

eNOS antibody was purchased from BD Transduction Laboratories (Lexington, KY, USA). Primary antibodies for ROCK-1, ROCK-2 and RhoA were provided by Santa Cruz Biotechnology, Inc (Santa Cruz, CA, USA). Horseradish peroxidase (HRP)conjugated secondary antibody was purchased from Pierce Biotechnology, Inc (Rockford, IL, USA). Rho activation kit for pull-down assay was purchased from Stressgen Bioreagent Corp. (Victoria, BC, Canada). Rho-kinase assay kit was supplied by CycLex Co., Ltd (Tera-Sawaoka Ina, Negano, Japan). Nitric oxide synthase assay kit was purchased from Cayman Chemical Europe, (Massy, France). Cyclic guanosine monophosphate (cGMP) enzyme immunoassay Biotrak (EIA) system was provided by GE Healthcare Life Sciences, (Little Chalfont, UK).
All other chemical products were purchased from SigmaAldrich (Steinheim, Germany).

\section{Measurement of isometric tension of pulmonary arterial vascular rings}

Each pulmonary arterial ring was mounted between two parallel stainless steel wires for measurement of isometric tension in organ chambers (Emka Technologies, Paris, France). Individual organ baths were filled with $20 \mathrm{~mL}$ of KrebsHenseleit solution (glucose $10 \mathrm{mM}$, pyruvate $2 \mathrm{mM}$, HEPES $10 \mathrm{mM}$, EDTA $0.03 \mathrm{mM}, \mathrm{NaCl} 118 \mathrm{mM}, \mathrm{KCl} 4.7 \mathrm{mM}, \mathrm{CaCl}_{2}$ $\left.2.5 \mathrm{mM}, \mathrm{KH}_{2} \mathrm{PO}_{4} 1.2 \mathrm{mM}, \mathrm{MgSO}_{4} 1.2 \mathrm{mM}, \mathrm{NaHCO}_{3} 15 \mathrm{mM}\right)$, heated at $37^{\circ} \mathrm{C}$ and continuously aerated with $95 \% \mathrm{O}_{2}$ and $5 \%$ $\mathrm{CO}_{2}$. The isometric force generated by the ring segment was measured and recorded by Macintosh Performa 630-Software (Apple). Pulmonary arterial rings were initially stabilised for 30 min between 1-1.5 g, at a resting tension that has been previously determined as corresponding to the optimal length for tension development.

The arterial rings were precontracted with increasing concentrations of phenylephrine $\left(10^{-8}-10^{-5} \mathrm{~mol} \cdot \mathrm{L}^{-1}\right)$ as previously described [16]. For each ring, the effective concentration of phenylephrine needed to achieve optimal contraction was calculated. Indomethacin $\left(10^{-5} \mathrm{~mol} \cdot \mathrm{L}^{-1}\right)$ was added to the organ baths $30 \mathrm{~min}$ prior to phenylephrine to exclude the influence of cyclooxygenase pathway products [17]. After the phenylephrine contraction series, the baths were washed three times with fresh Krebs solution and the rings were allowed to stabilise over a resting tension for $1 \mathrm{~h}$.

Endothelium-dependent relaxation was assessed by exposing pulmonary arterial rings to increasing concentrations of acetylcholine (Ach; $\left.10^{-9}-10^{-4} \mathrm{~mol} \cdot \mathrm{L}^{-1}\right)$. At least four rings from each patient were studied for obtained results.

\section{Immunohistochemistry assessment for eNOS, ROCK-1 and ROCK-2}

For eNOS immunohistochemical staining, sections were prepared as previously described to block nonspecific binding of primary and secondary antibodies and nonspecific reactions [18]. The sections were incubated with the specific eNOS antibody (1 in 100 titre). All bound antibody sections were incubated with HRP-conjugated secondary antibody. The sections were incubated with metal enhanced 3,3'-diaminobenzidine peroxidase substrate until the desired staining was obtained. Tissues were counterstained with haematoxylin and examined using light microscopy. Negative control sections were incubated in blocking buffer alone without primary antibody. eNOS immunostaining expression was measured in endothelial cells of pulmonary arterial rings. The staining expression was measured by using intensity score. At least ten counted images were performed for each patient.

For ROCK-1 and ROCK-2 immunostaining, sections were stained with ImmunoCruz ${ }^{\mathrm{TM}}$ staining system. Primary antibody (goat polyclonal immunoglobulin (Ig)G) was diluted to 1 in 50, as determined by titration in serum block. After $2 \mathrm{~h}$ of incubation with the primary antibody, sections were washed in PBS and incubated with biotinylated secondary antibody and HRP-streptavidin complex for $30 \mathrm{~min}$ each. The desired stain was obtained in $60 \mathrm{~s}$ after addition of HRP substrate mixture to 
the sections. The last steps with counterstained, dehydrated and mounted slides were then performed. The expression of ROCK-1 and ROCK-2 peroxidase staining was measured in SMCs of pulmonary arteries. Ten magnified visual fields were performed for each subject.

\section{Protein semi-quantification of eNOS, ROCK-1, ROCK-2, RhoA and p-MYPT-1 by Western blot}

Equal amounts of pulmonary arterial homogenates adjusted to protein content were subjected to $7.5 \%$ SDS-PAGE (for eNOS, ROCK-1, ROCK-2 and phosphorylated MYPT-1 (p-MYPT-1)) or $12 \%$ gels (RhoA) for electrophoresis and then transferred to PVDF membranes (Immobilon-P, Millipore SA, France). Membranes were blocked in 5\% non-fat milk, 0.1\% Tween-20 in PBS for $1 \mathrm{~h}$ at room temperature. The membranes were hybridised overnight with antibodies against eNOS (dilution 1 in 100), ROCKs (dilution 1 in 500), RhoA (dilution 1 in 100) and p-MYPT-1 (dilution 1 in 200), which were then incubated with HRP-linked goat anti-mouse (eNOS, dilution 1 in 5,000 ) or donkey anti-goat IgG-HRP secondary antibodies for $45 \mathrm{~min}$ for ROCKs (dilution 1 in 10,000), RhoA and p-MYPT-1 (dilution 1 in 5,000 ). The equal sample loadings were confirmed by $\beta$-actin Protein bands were developed on the film by using enhanced chemiluminescence reagent, according to the manufacturer's instructions. The protein densities were quantified by using Image Software System (Genius 2; Syngene, Cambridge, UK) and normalised with $\beta$-actin densities.

\section{eNOS activity}

The activity of eNOS was measured using a colorimetric NO synthase assay kit (Cayman Chemical Europe). This kit was used to evaluate the production of NO from L-arginine by the action of eNOS in the presence of nicotinamide adenine dinucleotide phosphate, an essential co-factor for this enzyme. The best index of total $\mathrm{NO}$ produced was the sum of both nitrite $\left(\mathrm{NO}_{2}^{-}\right)$and nitrate $\left(\mathrm{NO}_{3}^{-}\right)$concentration, which indirectly represented the activity of total eNOS. All steps of the assay were carried out according to the manufacturer's instructions. The final products of reaction were $\mathrm{NO}_{3}{ }^{-}$. The results were read at an absorbance of $540 \mathrm{~nm}$. The concentrations of $\mathrm{NO}_{3}{ }^{-}$from the samples were determined from the standard curve of the provided nitrate standard.

\section{cGMP concentration}

cGMP concentration in pulmonary arteries was measured by an enzyme immunoassay Biotrak (EIA) system (GE Healthcare Life Sciences) according to the manufacturer's instructions. It combined the use of a peroxidase-labelled cGMP conjugate, a specific antiserum that can be immobilised on pre-coated microplates, and a one-pot stabilised substrate solution. The assay was based on competition between unlabelled cGMP and a fixed quantity of cGMP labelled with peroxidise, for a limited number of binding sites on a cGMP specific antibody. Results were obtained by colorimetric reading with the absorbance at $450 \mathrm{~nm}$. The concentrations from the sample were quantified by interpolating absorbance readings from a standard curve generated with the calibration of provided cGMP protein standards.

\section{Measurement of RhoA active form by pull-down assay}

Rho activation was determined using a Rho activation kit (Stressgen Bioreagent Corp.). It was used to measure the active form of RhoA (GTP-RhoA). This assay used a glutathione $S$-transferase-fusion protein containing the Rho-binding domain of mouse rhotekin to affinity precipitate active Rho (GTP-Rho) from pulmonary arterial homogenates (500 $\mu \mathrm{g}$ total proteins). After the pull-down assay, $25 \mu \mathrm{L}$ of eluted samples were separated by $12 \%$ SDS-PAGE gels, transferred to PVDF membrane and probed with antibodies against RhoA. The protein bands were detected and quantified as described previously.

\section{Determination of the activity of Rho-kinase by ELISA}

Rho-kinase activity in pulmonary arteries was assessed by ELISA, using Rho-kinase assay kit (CycLex Co., Ltd). Plates were pre-coated with a substrate corresponding to the recombinant C-terminus of MBS (myosin-binding subunit of myosin phosphatase or MYPT-1), which contains a threonine residue that was phosphorylated by Rho-kinase. The specific detector antibody was HRP-conjugated anti-phospho-MBS (AF20) that detected only the phosphorylated form of threonine-696 on MBS. The amount of phosphorylated substrate was measured by binding it with a HRP of AF20 which then catalysed the conversion of the chromogenic substrate tetra-methylbenzidine from a colourless solution. It was then quantified by spectrophotometry and reflected the relative amount of Rho-kinase activity on phosphorylation of MYPT in pulmonary arterial SMC.

\section{Statistical analysis}

Results are presented as mean \pm SEM. The software SPSSversion 13.0 (SPSS, Chicago, IL, USA) was used for all statistical analysis. Comparisons between groups for characteristics were performed with the unpaired t-test. Comparisons of dose-response curves for relaxation response were analysed by ANOVA for repeated measure. Values of $p<0.05$ were considered statistically significant.

\section{RESULTS}

\section{Baseline clinical and functional characteristics}

All 16 subjects (eight nonsmokers and eight smokers) had normal lung function test. Their clinical and functional characteristics are shown in table 1.

TABLE 1 Patients' clinical and functional characteristics

\begin{tabular}{|c|c|c|}
\hline Variables & Nonsmokers & Smokers \\
\hline Patients $\mathbf{n}$ & 8 & 8 \\
\hline Age yrs & $65 \pm 11$ & $55 \pm 8$ \\
\hline Male/female $\mathbf{n}$ & $1 / 7$ & $7 / 1$ \\
\hline Tobacco pack-yrs & & $37 \pm 11$ \\
\hline FEV1 L & $2.47 \pm 0.49$ & $2.81 \pm 0.55$ \\
\hline FEV $1 \%$ pred & $110 \pm 26$ & $96 \pm 5$ \\
\hline FEV $_{1} /$ FVC $\%$ pred & $81 \pm 5$ & $78 \pm 4$ \\
\hline $\mathrm{Pa}, \mathrm{O}_{2} \mathrm{mmHg}$ & $86 \pm 8$ & $83 \pm 9$ \\
\hline $\mathrm{Pa}, \mathrm{CO}_{2} \mathrm{mmHg}$ & $36 \pm 4$ & $39 \pm 4$ \\
\hline
\end{tabular}

Data are presented as mean \pm SEM, unless otherwise stated. FEV 1 : forced expiratory volume in $1 \mathrm{~s}$; FVC: forced vital capacity; \% pred: \% predicted; $\mathrm{Pa} \mathrm{O}_{2}$ arterial oxygen tension; $\mathrm{Pa}, \mathrm{CO}_{2}$ : arterial carbon dioxide tension. 


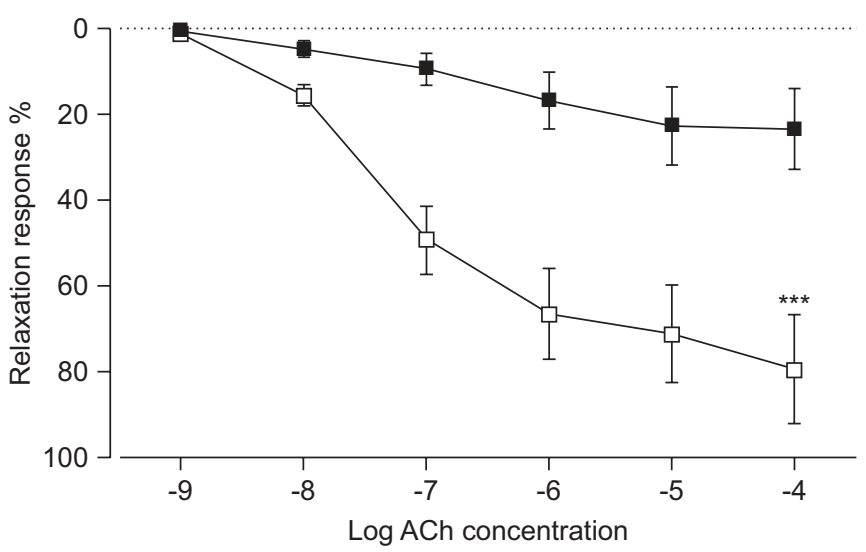

FIGURE 1. Endothelium-dependent relaxation of pulmonary arterial rings. Endothelium-dependent relaxation in response to acetylcholine (ACh; concentration in $\mathrm{mol} \cdot \mathrm{L}^{-1}$ ) of pulmonary arterial rings from nonsmokers and smokers with normal lung function. Values are presented as mean \pm SEM. $n=8$ for each group $\square$ : nonsmokers; $\mathbf{\square}$ : smokers; ${ }^{* * *}: \mathrm{p}<0.001$

\section{Endothelium-dependent relaxation in response to $\mathrm{ACh}$}

Endothelium-dependent pulmonary arterial relaxation in response to ACh $\left(10^{-9}-10^{-4} \mathrm{~mol} \cdot \mathrm{L}^{-1}\right)$ was significantly reduced in smokers compared with controls ( $\mathrm{p}<0.001$; fig. 1).

\section{eNOS protein expression}

Semi-quantitative analysis for positive peroxidase staining and protein density of eNOS expression by Western blotting in pulmonary arteries showed no significant difference between smoker and nonsmoker subjects ( $\mathrm{p}>0.05$; fig. $2 \mathrm{a}-\mathrm{c})$.

\section{Concentration of $\mathrm{NO}_{3}{ }^{-}$and $\mathrm{CGMP}$}

The $\mathrm{NO}_{3}{ }^{-}$concentration in pulmonary arterial homogenates of smokers was significantly lower than that in nonsmokers $(p<0.01)$. Similarly, the concentration of cGMP obtained from pulmonary arterial homogenates of smokers was significantly lower compared with nonsmokers ( $p<0.01$; fig. $2 d$ ).

\section{ROCK-1 and ROCK-2 immunostaining and protein expression}

Peroxidase staining scores and protein densities showed that expression of ROCK-1 (fig. 3a-c) and ROCK-2 (fig. 3d-f) in pulmonary arteries of smokers were significantly higher than nonsmokers $(p<0.01)$.

\section{GTP-RhoA and total RhoA protein expression}

Western blot analysis of total RhoA protein in pulmonary arteries showed no difference between the two groups ( $\mathrm{p}>0.05$; fig. 4). Results of a pull-down assay showed GTPRhoA protein expression in smokers was significantly higher than in nonsmokers ( $p<0.01$; fig. 4). Activity of RhoA in pulmonary arteries, as assessed by the ratio of GTP-RhoA/ total RhoA, was significantly higher in smokers as compared with nonsmoker subjects ( $\mathrm{p}<0.01$; fig. 4 ).

\section{p-MYPT-1 protein expression}

p-MYPT1 protein density measured by Western blot was significantly increased in smokers in comparison with nonsmokers $(\mathrm{p}<0.01$; fig. 5). The concentration of $\mathrm{p}-\mathrm{MYPT}-1$, quantified by a)

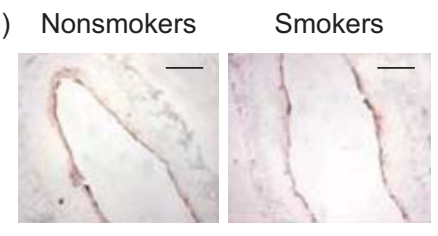

b)

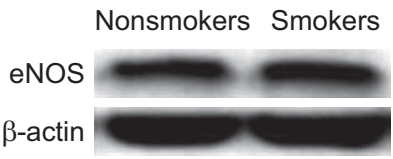

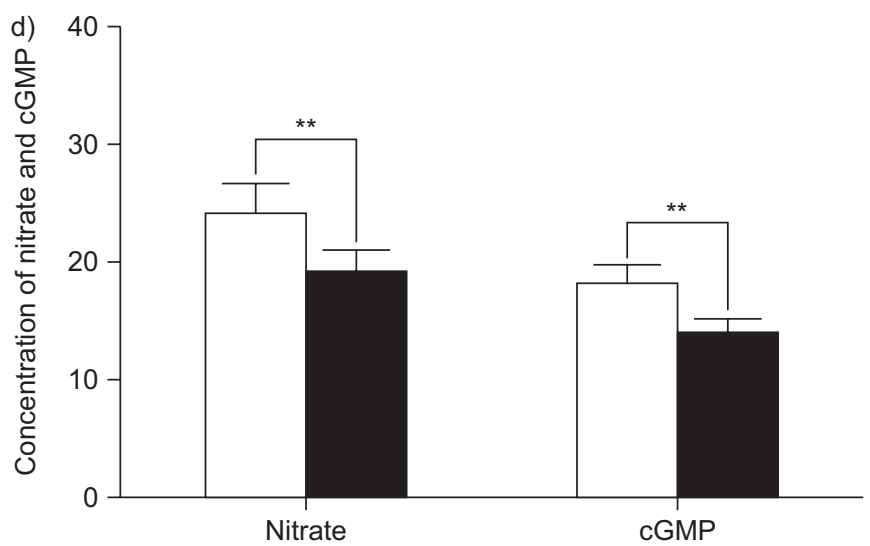

FIGURE 2. Expression of endothelial nitric oxide synthase (eNOS) in nonsmokers and smokers. a) Endothelial pulmonary arteries stained with eNOS and b) samples of eNOS protein expression measured by Western blot. Scale bars $=200 \mu \mathrm{m}$. c) eNOS protein expression measured by immunohistochemistry and Western blot in pulmonary arteries of nonsmokers ( $\square$ ) and smokers Protein density is presented as eNOS/ $\beta$-actin ratio. NS: no significant difference $\mathrm{n}=8$ for each group. d) Concentration of nitrate $\left(\mu \mathrm{mol} \cdot \mathrm{mL}^{-1}\right)$ and cyclic guanosine monophosphate (cGMP; $\left.\mu \mathrm{g} \cdot \mathrm{L}^{-1} \times 0.1\right)$ measured by ELISA from pulmonary arterial homogenates of nonsmokers $(\square)$ and smokers $(\mathbf{\square})$. Values are presented as mean \pm SEM. $n=8$ for each group. ${ }^{* *}: p<0.01$.

ELISA, showed it was higher in smokers compared with nonsmokers ( $p<0.001$; fig. 5).

\section{DISCUSSION}

The deleterious effect of cigarette smoke on endothelial function has already been reported in the systemic circulation of asymptomatic smokers $[6,7,11]$. Only a few reports have to date investigated the underlying mechanisms of pulmonary endothelial dysfunction in smokers with mild COPD $[9,10]$ or normal lung function [19]. The main results of the present study, assessing pulmonary endothelial function of smokers with normal lung function as compared with nonsmokers, 
a)
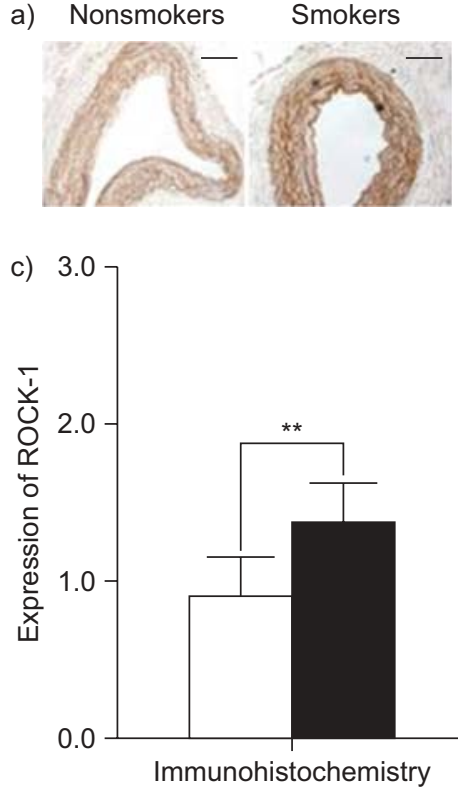

d)

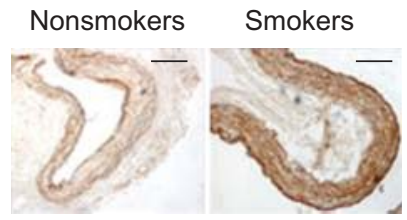

f) 3.0

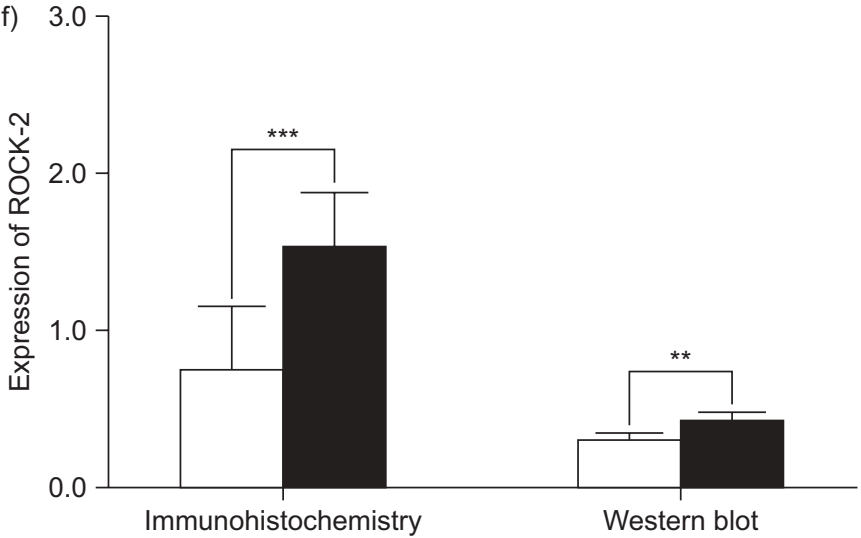

FIGURE 3. Expression of Rho-kinase (ROCK)-1 in nonsmokers and smokers. a) Smooth muscle cells of pulmonary arteries stained with ROCK-1 and b) samples of ROCK-1 protein expression measured by Western blot. C) ROCK-1 protein expression measured by immunochemistry and Western blot from the pulmonary arteries of nonsmokers $(\square)$ and smokers ( $\square)$. Protein density is presented as ROCK-1/ $\beta$-actin ratio. $n=8$ for each group. d) Smooth muscle cells of pulmonary arteries stained with ROCK-2 and e) samples of ROCK-2 protein expression measured by Western blot. f) ROCK-2 protein expression measured by immunohistochemistry and Western blot from pulmonary arteries of nonsmokers $(\square)$ and smokers ( $\square$ ). Protein density is presented as ROCK-2/ $\beta$-actin ratio. $n=8$ for each group. Values are presented as mean \pm SEM. Scale bars $=200 \mu \mathrm{m}$. ${ }^{*}$ : $p<0.01$; $* * *: p<0.001$

can be summarised as follows: 1) pulmonary endotheliumdependent relaxation in response to $\mathrm{ACh}$ was significantly impaired in smokers; 2) this impairment was associated with reduced activity but not expression of eNOS; together with 3)
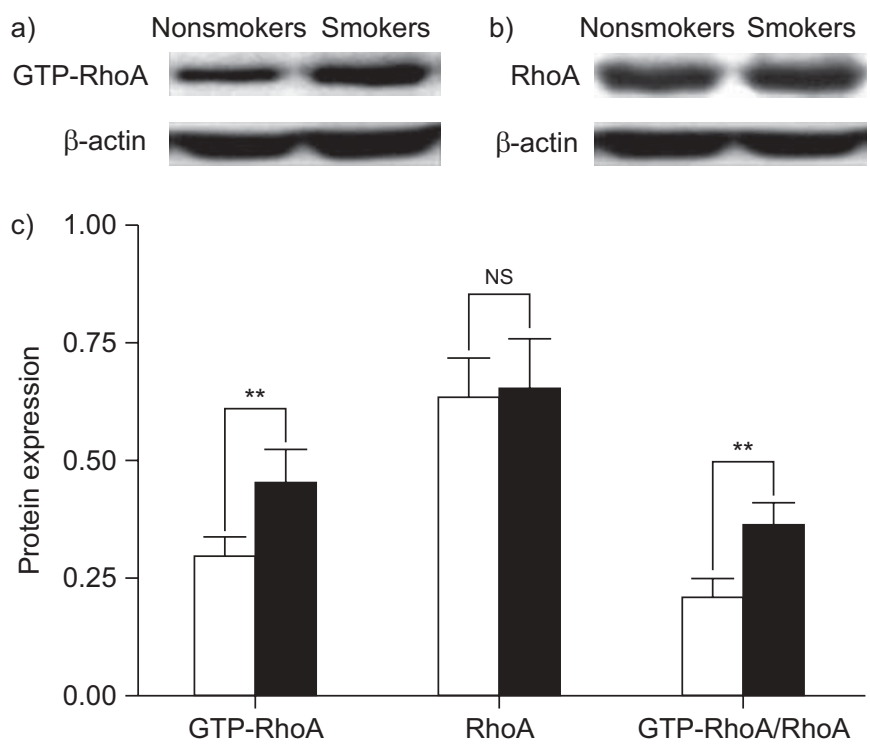

FIGURE 4. Expression of guanosine triphosphate (GTP)-RhoA and RhoA in nonsmokers and smokers. Samples of a) GTP-RhoA and b) RhoA protein expression. C) Protein expression of GTP-RhoA measured by pull-down assay and RhoA measured by Western blot from pulmonary arterial homogenates of nonsmokers $(\square)$ and smokers $(\boldsymbol{\square})$. Protein density is presented as GTP-RhoA/ $\beta$ actin and RhoA/B-actin ratio. GTP-RhoA/total RhoA presented as RhoA activity. Values are presented as mean \pm SEM. NS: no significant difference. $n=8$ for each group. ${ }^{* *}: p<0.01$

increased activity and expression of the Rho-kinases (ROCK-1 and ROCK-2).

If the results of the present study showing significant impairment of pulmonary arterial relaxation in response to ACh in smokers with normal lung function have not yet been reported, several studies have previously suggested the possibility of endothelial dysfunction in both the systemic $[6,7,11]$ and pulmonary circulation [10] of healthy smokers. This observation is consistent with the hypothesis that pulmonary endothelial dysfunction not only occurs in patients with end-stage lung disease [20], but it can also be seen in patients with milder degrees of COPD [5, 9, 10], and in supposedly healthy smokers [10, 19]. As endothelial dysfunction is not restricted to the pulmonary circulation, it is likely that the underlying mechanisms are related to systemic oxidative stress caused by tobacco smoking [21, 22].

Decreased production and/or reduced bioavailability of NO might account for endothelial dysfunction [8, 11, 19]. In the present study, protein expression of eNOS did not significantly differ (fig. 2a-c), but eNOS activity, as assessed by nitrate measurement and the concentration of cGMP (fig. 2d) were significantly reduced in smokers compared with nonsmokers. Thus, impairment of relaxation is probably not due to reduced eNOS expression but rather to impaired eNOS activity (nitrate production) and NO bioavailability (cGMP concentration) in pulmonary arteries from smokers. Results from the present study differed from previous reports showing impaired eNOS expression in pulmonary arteries from healthy smokers [19]. The reduced activity of eNOS found in the present study, however, is consistent with all previous findings showing 

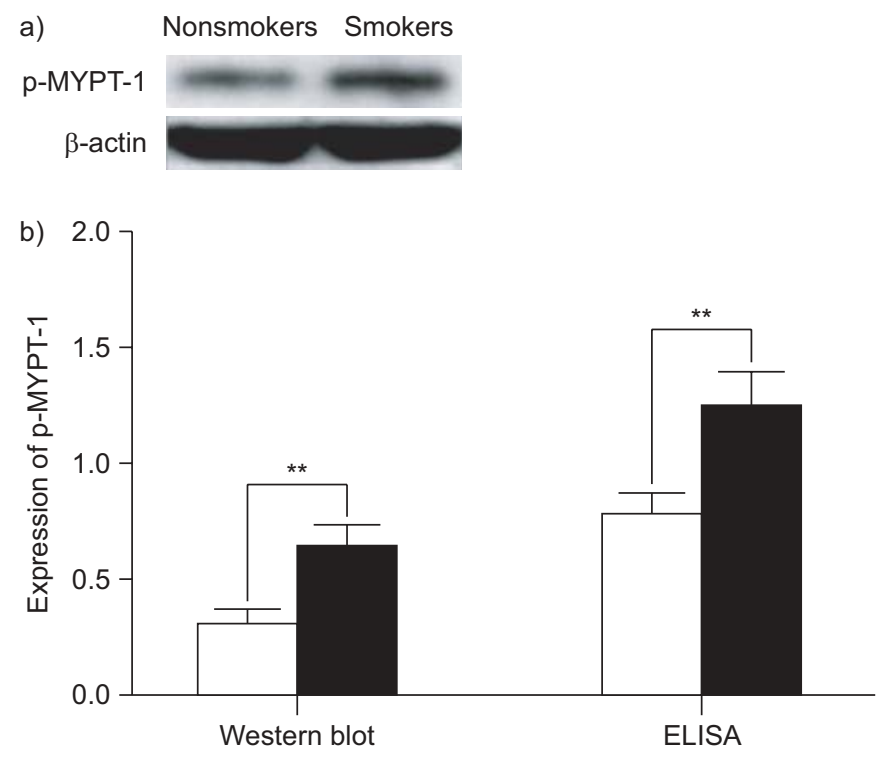

FIGURE 5. Expression of phosphorylated myosin phosphatase subunit 1 ( $p$-MYPT-1) in nonsmokers and smokers. a) Samples of p-MYPT-1 protein expression measured by Western blot. b) p-MYPT-1 protein expression measured by Western blot and ELISA from pulmonary arterial homogenates of nonsmokers ( $\square$ ) and smokers ( $\mathbf{\square})$. Protein density measured by Western blot is presented as p-MYPT-1/ $\beta$-actin. Values are presented as mean \pm SEM. $n=8$ for each group. ${ }^{*}$ : $p<0.01$.

impaired NO-mediated pulmonary relaxation in smokers either without [19], or with moderate [9, 10] or severe [20] lung disease.

The role of the RhoA/Rho-kinase pathway in causing impairment of endothelium-dependent relaxation has been recently demonstrated in the systemic circulation of smokers [12]. Augmented Rho-kinase activity in peripheral leukocytes is related to impaired flow-mediated vasodilatation [23] and increased aortic stiffness [24] in cigarette smokers. There is mounting evidence suggesting important and complex crosstalk between RhoA/Rho-kinase and the NO/cGMP pathways. Rho-kinases can downregulate eNOS expression and activity, and NO bioavailability [25], whereas eNOS mRNA stability is increased by Rho-kinase inhibitors [26]. The latter can, furthermore, negate the inhibition of Rho-kinases on Akt, a protein kinase that stimulates eNOS activity through a calcium-independent pathway, thus leading to increase in NO production [27, 28].

Together with protein expression of Rho-kinases, the active form of RhoA (RhoA-GTP) and the activity of Rho-kinase (as assessed by measurement of the phopshorylated form of MYPT-1) have also been evaluated in the present study. The results showed that impairment of endothelium-dependent relaxation in smokers was associated with significant increase of expression of RhoA-GTP (fig. 4) and p-MYPT-1 (fig. 5). As the phosphorylation of MYPT-1 caused by Rho-kinases results in the inhibition of MPT, thereby sustaining actin-myosin interaction and prolongs pre-existing vasoconstriction [13], it is conceivable that increased RhoA/Rho-kinase activity further aggravates the imbalance between pulmonary vasoconstrictors and dilators towards increased vascular tone in the pulmonary circulation of healthy smokers. Consistent with the central role of Rho-kinases in the modulation of pulmonary vascular tone is the recent demonstration that fasudil, a Rho-kinase inhibitor, significantly improved experimental pulmonary arterial hypertension to a larger extent than endothelin receptors antagonists and inhibitors of phosphodiesterase 5 [29].

\section{Conclusion}

Limitations of the present study include the relative small number of patients with different male/female ratios in each study group, and the lack of assessment of endotheliumdependent relaxation in the presence of the Rho-kinase inhibitors. Our study has nevertheless provided evidence linking pulmonary endothelial dysfunction in smokers with normal lung function and the reduction of eNOS activity, related to the upregulation of the RhoA/Rho-kinase pathway. The interaction between the RhoA/Rho-kinase pathway and eNOS/NO signalling in smokers should be investigated.

\section{STATEMENT OF INTEREST}

None declared.

\section{REFERENCES}

1 Pope CA 3rd., Burnett RT., Krewski D., et al, Cardiovascular mortality and exposure to airborne fine particulate matter and cigarette smoke: shape of the exposure-response relationship. Circulation 2009; 120: 941-948.

2 Silverman EK, Speizer FE. Risk factors for the development of chronic obstructive pulmonary disease. Med Clin North Am 1996; 80: 501-522.

3 Hogg JC. Pathophysiology of airflow limitation in chronic obstructive pulmonary disease. Lancet 2004; 364: 709-721.

4 Chaouat A, Naeije R, Weitzenblum E. Pulmonary hypertension in COPD. Eur Respir J 2008; 32: 1371-1385.

5 Santos S, Peinado VI, Ramirez J, et al. Characterization of pulmonary vascular remodelling in smokers and patients with mild COPD. Eur Respir J 2002; 19: 632-638.

6 Celermajer DS, Sorensen KE, Georgakopoulos D, et al. Cigarette smoking is associated with dose related and potentially reversible impairment of endothelium-dependent dilation in healthy young adults. Circulation 1993; 88: 2149-2155.

7 Celermajer DS, Adams MR, Clarkson P, et al. Passive smoking and impaired endothelium dependent arterial dilation in healthy young adults. N Engl J Med 1996; 334: 150-154.

8 Dinh-Xuan AT. Endothelial modulation of pulmonary vascular tone. Eur Respir J 1992; 5: 757-762.

9 Peinado VI, Barberà JA, Ramirez J. Endothelial dysfunction in pulmonary arteries of patient with COPD. Am J Physiol 1998; 274: 908-913.

10 Peinado VI, Barberà JA, Abate $\mathrm{P}$, et al. Inflammatory reaction in pulmonary muscular arteries of patients with mild chronic obstructive pulmonary disease. Am J Respir Crit Care Med 1999; 159: 1605-1611.

11 Barua RS, Ambrose JA, Eales-Reynolds LJ, et al. Dysfunctional endothelial nitric oxide biosynthesis in healthy smokers with impaired endothelium-dependent vasodilatation. Circulation 2001; 104: 1905-1910.

12 Noma K, Goto C, Nishioka K, et al. Smoking, endothelial function, and Rho-kinase in humans. Arterioscler Thromb Vasc Biol 2005; 25 2630-2635.

13 Loirand G, Guérin P, Pacaud P. Rho kinases in cardiovascular physiology and pathophysiology. Circ Res 2006; 98: 322-334.

14 Ming XF, Viswambharan H, Barandier C, et al. Rho GTPase/Rho kinase negatively regulates endothelial nitric oxide synthase 
phosphorylation through the inhibition of protein kinase B/Akt in human endothelial cells. Mol Cell Biol 2002; 22: 8467-8477.

15 Rabe KF, Hurd S, Anzueto A, et al. Global strategy for the diagnosis, management, and prevention of chronic obstructive pulmonary disease: GOLD executive summary. Am J Respir Crit Care Med 2007; 176: 532-555.

16 Cherry PD, Gillis CN. Evidence for the role of endothelium derived relaxing factor in acetylcholine-induced vasodilatation in the intact lung. J Pharmacol Exp Ther 1988; 247: 42-46.

17 Davenpeck KL, Guo JP, Lefer AM. Pulmonary artery endothelial dysfunction following ischemia and reperfusion of the rabbit lung. Circ Res 1989; 65: 1508-1515.

18 Hoehn T, Preston AA, McPhaden AR, et al. Endothelial nitric oxide synthase (NOS) is upregulated in rapid progressive pulmonary hypertension of the newborn. Intensive Care Med 2003; 29: 1757-1762.

19 Barberà JA, Peinado VI, Santos S, et al. Reduced expression of endothelial nitric oxide synthase in pulmonary arteries of smokers. Am J Respir Crit Care Med 2001; 164: 709-713.

20 Dinh-Xuan AT, Higenbottam TW, Clelland CA, et al. Impairment of endothelium-dependent pulmonary-artery relaxation in chronic obstructive lung disease. N Engl J Med 1991; 324: 1539-1547.

21 Raij L, DeMaster EG, Jaimes EA. Cigarette smoke-induced endothelium dysfunction: role of superoxide anion. J Hypertens 2001; 19: 891-897.
22 van der Vaart H, Postma DS, Timens W, et al. Acute effects of cigarette smoke on inflammation and oxidative stress: a review. Thorax 2004; 59: 713-721.

23 Hidaka T, Hata T, Soga J, et al. Increased leukocyte Rho kinase (ROCK) activity and endothelial dysfunction in cigarette smokers. Hypertens Res 2010; 33: 354-359.

24 Noma K, Goto C, Nishioka K, et al. Roles of Rho-associated kinase and oxidative stress in the pathogenesis of aortic stiffness. J Am Coll Cardiol 2007; 49: 698-705.

25 Laufs U, Endres M, Liao JK. Regulation of endothelial NO production by Rho GTPase. Med Klin 1999; 94: 211-218.

26 Laufs U, Liao JK. Post-transcriptional regulation of endothelial nitric oxide synthase mRNA stability by Rho GTPase. J Biol Chem 1998; 273: 24266-24271.

27 Wolfrum S, Dendorfer A, Rikitake Y, et al. Inhibition of Rho-kinase leads to rapid activation of phosphatidylinositol 3-kinase/protein kinase Akt and cardiovascular protection. Arterioscler Thromb Vasc Biol 2004; 24: 1842-1847.

28 Ming XF, Viswambharan H, Barandier C, et al. Rho GTPase/Rho kinase negatively regulates endothelial nitric oxide synthase phosphorylation through the inhibition of protein kinase B/Akt in human endothelial cells. Mol Cell Biol 2002; 22: 8467-8477.

29 Mouchaers KT, Schalij I, de Boer MA, et al. Fasudil reduces monocrotaline-induced pulmonary arterial hypertension: comparison with bosentan and sildenafil. Eur Respir J 2010; 36: 800-807. 